\title{
THE METHODOLOGY FOR DEVELOPING THE KINEMATIC MODEL OF SELECTED CPR-A SYSTEM AS A NECESSITY FOR THE DEVELOPMENT OF A DYNAMIC MODEL
}

\author{
Dr Mirjana Filipović* \\ Mihajlo Pupin Institute, Belgrade, Serbia \\ Ana Đurić \\ Wayne State University, Detroit, USA \\ Ljubinko Kevac \\ School of Electrical Engineering, Belgrade, Serbia
}

The authentic form or general form of Cable Suspended Parallel Robot type A, CPR-A mathematical model is defined. The proper definition of the system kinematic model which includes trajectory, velocity and acceleration is a prerequisite for the formulation of a dynamic model. These three components represent the basic functional criteria of the real system which is described by the corresponding geometric relations and differential equations. Kinematic model is defined for the CPR-A system via the Jacobian matrix. An adequate choice of generalized coordinates (in this paper, the motor coordinates), provides a mathematical model that illuminates the mapping of motor (resultant forces in the ropes) and camera carrier forces (acting on a camera carrier) over the Jacobian matrix on motion dynamics of each motor. Software packages AIRCAMA (aerial camera system type A) are formed and used for individual and comparative analysis of the CPR-A system from various aspects. The impact of changing any parameter of the system (workspace dimensions, the mass of a camera carrier, change the size and dynamics of power disturbances, the choice of control law, the reference trajectory, and the presence of singularity avoidance system and a number of other characteristics) can be analyzed through these software packages. Different examples of the CPR-A system motion are analyzed and their results are presented. Application possibilities of CPR-A system are certainly much broader than it may be assumed at this moment, especially for sports, cultural, military or police purposes.

Key words: Cable-suspended parallel robot, Observation, Workspace, Modeling, Kinematic, Dynamic

\section{INTRODUCTION}

A system for observation of the workspace with moving objects has been developed to some extent and widely analyzed in various research areas as well as for different applications. Similar systems were analyzed and modeled as presented by numerous publications.

The kinematic design of a planar three-degreeof-freedom parallel manipulator is considered, in paper [13]. Four optimal different design criteria are established and analyzed. A trajectory planning approach for cable-suspended parallel mechanisms has been presented in [14]. A planar two-degree-of-freedom parallel mechanism has been used in the analysis. Paper [03] studied the kinematics and statics of under- constrained cable-driven parallel robots with less than six cables, in crane configuration. In those robots, kinematics and statics are intrinsically coupled and they must be dealt with simultaneously. A motion controller for six DOF tendon-based parallel manipulator (driven by seven cables), which moves a platform with high speed is introduced in [08]. The Workspace conditions and the dynamics of the manipulator are described in details. In the paper [01] authors presented algorithms that enable precise trajectory control of the Networked Info Mechanical Systems - NIMS, and under constrained threedimensional $-3 D$ cabled robot intended for use in actuated sensing. They provide a brief system overview and then describe methods to determine the range of operation of the robot. Sev- 
eral prototypes of the wire-driven parallel robots, with different actuation schemes have been presented in [22]. Two of them have been evaluated through extensive tests and showed unexpected kinematic problems. The wrench-closure workspace of parallel cable-driven mechanisms is the set poses of their mobile platform for which the cables can balance any external wrench. The determination of this workspace is an important issue in [15] since the cables can only pull and not push on the mobile platform. Parallel cabledriven Stewart-Gough platforms consist of an end-effector which is connected to the machine frame by motor driven cables. Since cables can transmit only tension forces, at least $m=n+1$ cables are needed to tense a system having $n$ degrees-of-freedom. This will cause a kinematical redundancy and leads to a ( $m$ - n)-dimensional solution space for the cable force distribution presented in [02]. The recent result from a newly designed parallel wire robot which is currently under construction is presented in [27]. It has been used for developing a new technique for computation and transfer of its workspace to the available CAD software. An auto-calibration method for over constrained cable-driven parallel robots using internal position sensors located in the motors has been presented in [23]. A calibration workflow is proposed and implemented including pose selection, measurement, and parameter adjustment. The wire-driven parallel robot presented in [18] has attracted the interest of researchers since the very beginning of the study of parallel robots. This type of robot has the advantage of having light mobile mass, simple linear actuators with possibly relatively large stroke and less risk of interference between the legs. On the other hand their major drawback is that wire actuator can only pull and not push. The paper [26] addressed the issue of control design for a redundant 6-DOF cable robot with positive input constraints. The design is based on feedback linearization controller named reference governor - RG. Nonlinear dynamic analysis of the suspended cable system is carried out with some sensible results presented in [07] that could be useful to the real engineering of LSRT (Large Spherical Radio Telescopes). Integrated mechanical, electronic, optic and automatic control technologies are employed to make considerable improvement upon the same system. A multiple cable robotic crane designed in [31] is used to provide improved cargo handling. The equations of motion are derived for the cargo and flexible cable using Lagrange's equations and assumed modes method. The results are compared against desired cable lengths and results achieved in previous research using a rigid cable model. This is one of a few papers dealing with flexible ropes. For the requirement of the trajectory tracking of the LSRT, a large fine tuning platform based on the Stewart platform is presented in papers [32] and [33]. The mathematical model for kinematic control is developed with coordinate transformation, and dynamic analysis is made using a Jacobian matrix, which, with singularity analysis, built a solid base for the tracking control. Paper [20] addresses the static analysis of the cable-driven robotic manipulators with the non-negligible cable mass. An approach to computing the static displacement of a homogeneous elastic cable is presented. The resulting cable-displacement expression is used to solve the inverse kinematics of general cable-driven robotic manipulators. Cable suspended parallel robot is analyzed in [35], in which cables are utilized to replace links to manipulate objects. It is developed from parallel and serial cable-driven robot. Compared with the parallel robot, this type of robot has more advantages. The cooperative variation of lengths of six cables pulls the feed cabin to track radio source with six degrees of freedom. The cable-driven parallel manipulator can only bear tension, but not compression. Therefore, a cable system with j end-effectors DOFs requires at least $(j+1)$ cables as shown in [19]. For three-translational motions of the feed in the system, a four-cable-driven parallel manipulator has been developed. For the design of the five-hundred-meter aperture spherical radio telescope, a four-cable-driven parallel manipulator, which is long in span and heavy in weight, is adopted as the first-level adjustable feed-support system. The goal of the paper [34] is to optimize dimensions of the four-cable-driven parallel manipulator to meet the workspace requirement of constraint condition in terms of cable tension and stiffness. One of the works that deals with visualization of workspace is presented in [6].

In the current literature survey there is no any mathematical model of the cable-suspendedparallel robots with double parallel ropes and there is no available procedure for generating the Jacobian matrix of the similar systems. Another problem is that the current published models do not include the motors dynamics. The analysis 
and synthesis of these complex systems cannot be proper without the motor dynamics, because it represents the dominant dynamics for any electro mechanical system. The kinematic formulation of the presented CPR-A system is a key contribution in this paper, which will be used for the system realization. This formulation can be used for determining the Jacobian matrix of any CPR system configuration. This methodology for developing the kinematic model of selected CPR systems is named the KinCPR-Solver (Kinematic Cable Parallel Robot Solver), and it gives a precise direct and inverse kinematic solutions. The dynamic model is generated using the fundamental dynamic theory based on the Lagrange's principle of virtual work. The mathematical model of the motors is determined using the same equations and expressed with the generalized coordinates.

In this paper the camera carrier workspace has the shape of a parallelepiped, such that the camera carrier hangs over the ropes properly connected on the four highest points i.e. the four upper angles of the workspace. The suspension system is defined in these four points. This paper analyzes the mechanism which involves only the positioning of the camera carrier. The camera motion is controlled by the ropes which are synchronized by three motors. The gyro sensor, which is installed in the camera carrier, is stabilized towards the horizon. In this paper, the real system abstraction will be used for modeling purposes by neglecting the transverse vibrations of the ropes (un-stretchable ropes).

Future researches include implementation of the features of elastic ropes (type of nonlinear dynamic elasticity as defined in [09]-[12], [16]-[17], [28]-[30], ) in the mathematical model of the CPR. In future research several different CPR models will be unified according to their similarities into one reconfigurable model, using the approaches presented in [04] and [05].

Most of the Section 2 is devoted to the CPR-A kinematic model, which is directly involved in the development of its dynamic model. Several cases of the system responses are analyzed for different conditions in the Section 3. In the Section 4 the concluding remarks are presented.

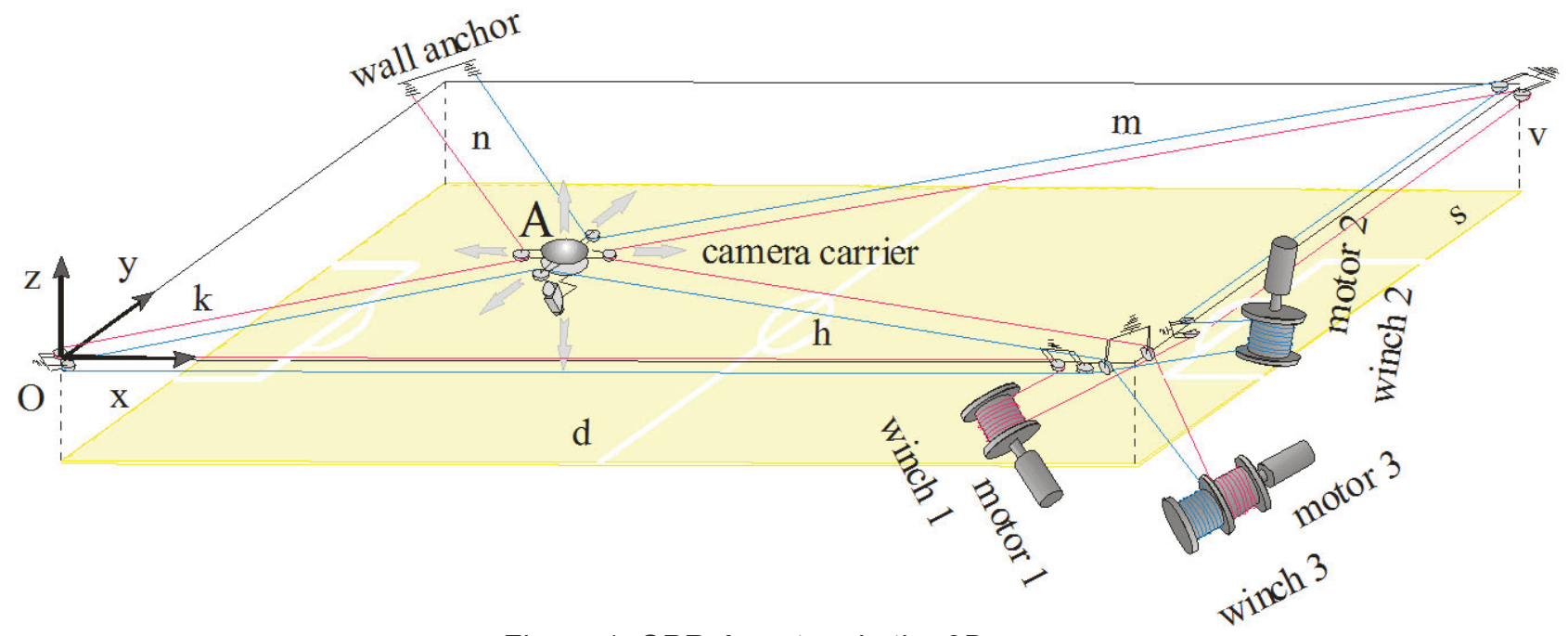

Figure 1: CPR-A system in the $3 D$ space

\section{MATHEMATICAL MODEL OF THE CABLE SUSPENDED PARALLEL ROBOT}

In this Section chosen CPR-A system is analyzed in depth, which graphical representation is shown in Figure 1 and Figure 2.

The camera carrier of the CPR-A structure is guided through the work area of the parallelepiped shape with two ropes connected with three winches, each powered by the motors.

The ropes of the pulley system are run on the winches (reel) 1, 2, 3, powered by the motors. The ropes coil or uncoil on the winches of radius $R_{1}, R_{2}$, and $R_{3}$. The motors rotate winches directly and its angular positions are $\theta_{1}, \theta_{2}$, and $\theta_{3}$. This motion moves the camera in the $x, y, z$ Cartesian coordinates.

To define a dynamic model of the CPR-A for observation of moving objects in workspace depicted in Figure1, it is first necessary to define the camera carrier velocity of change of the coordinates $\overrightarrow{\dot{p}}=\left[\begin{array}{lll}\dot{x} & \dot{y} & \dot{z}\end{array}\right]^{T}$ and the velocity of change 
of the motor coordinates $\overrightarrow{\dot{\phi}}=\left[\begin{array}{lll}\dot{\theta}_{1} & \dot{\theta}_{2} & \dot{\theta}_{3}\end{array}\right]^{T}$. The geometrical relationship between the lengths $k$, $h, m, n$ and the Cartesian coordinates position $x, y, z$ of the camera carrier is defined by the following equations:

$$
\begin{aligned}
& k=\sqrt{x^{2}+y^{2}+z^{2}} \\
& h=\sqrt{(d-x)^{2}+y^{2}+z^{2}} \\
& m=\sqrt{(d-x)^{2}+(s-y)^{2}+z^{2}} \\
& n=\sqrt{x^{2}+(s-y)^{2}+z^{2}}
\end{aligned}
$$

In Figure 3a, the motion of the motor 1 and the motor 2 are depicted. Both motors motion depend on their angular positive directions, which produce winding or unwinding of the ropes.

The motor 1 and the motor 2 motions toward the wall anchors (we call this line "before" motor) are expressed with the following equations respectively:

$$
\begin{aligned}
& \frac{\Delta \theta_{1}}{\Delta t} \cdot R_{1}=\frac{\Delta k}{\Delta t}+\frac{\Delta n}{\Delta t} \\
& \frac{\Delta \theta_{2}}{\Delta t} \cdot R_{2}=\frac{\Delta m}{\Delta t}+\frac{\Delta n}{\Delta t}
\end{aligned}
$$

The third motor is used to wind up the two ropes about the coil 3 . Those motion produce winding or unwinding of both ropes at the same time. This can be seen in Figure 2 and 3a).

The winch used for winding ropes has radius $R_{3}$. The relation between the third motor motion changes $\Delta \theta_{3}$ and the lengths change $\Delta k, \Delta h, \Delta m, \Delta h$, are expressed in the equations (7) and (8).

$\frac{\Delta \theta_{3}}{\Delta t} \cdot R_{3}=\frac{\Delta h}{\Delta t}+\frac{\Delta k}{\Delta t}+\frac{\Delta \theta_{2}}{\Delta t} \cdot R_{2}$

Or it can be expressed as following:

$$
\frac{\Delta \theta_{3}}{\Delta t} \cdot R_{3}=\frac{\Delta h}{\Delta t}+\frac{\Delta m}{\Delta t}+\frac{\Delta \theta}{\Delta t} \cdot R_{1}
$$

The equation (9) is obtained by substituting (6) into the equation (7) or (5) into the equation (8):

$\frac{\Delta \theta_{3}}{\Delta t} \cdot R_{3}=+\frac{\Delta k}{\Delta t}+\frac{\Delta h}{\Delta t}+\frac{\Delta m}{\Delta t}+\frac{\Delta n}{\Delta t}$

If the sampling time $\Delta t$ is small enough then the equations (5), (6), and (9) can be expressed, respectively, as:

$$
\begin{aligned}
& \dot{\theta}_{1} \cdot R_{1}=\dot{k}+\dot{n} \\
& \dot{\theta}_{2} \cdot R_{2}=\dot{m}+\dot{n} \\
& \dot{\theta}_{3} \cdot R_{3}=+\dot{k}+\dot{h}+\dot{m}+\dot{n}
\end{aligned}
$$

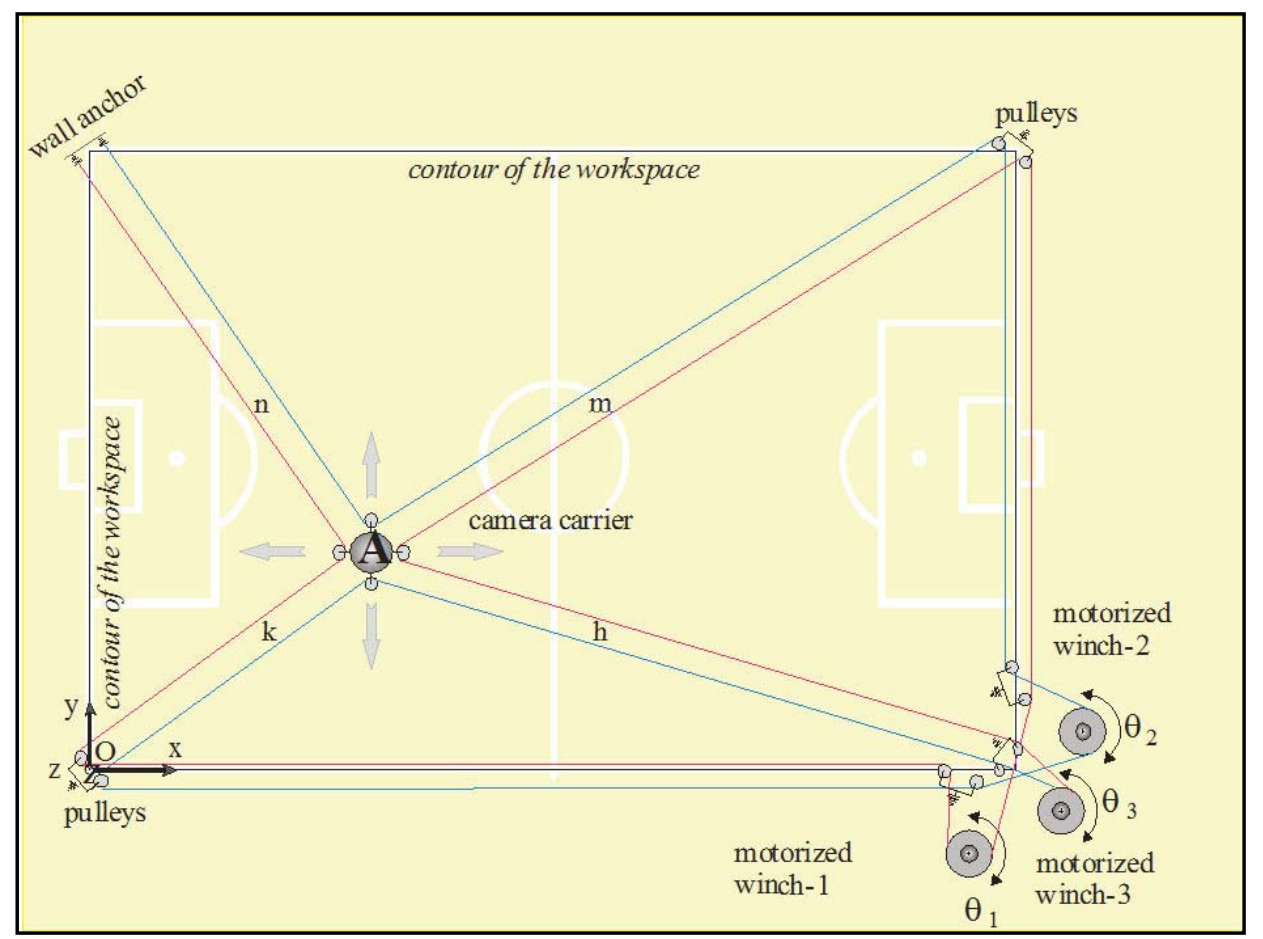

Figure 2: CPR-A system top view. 


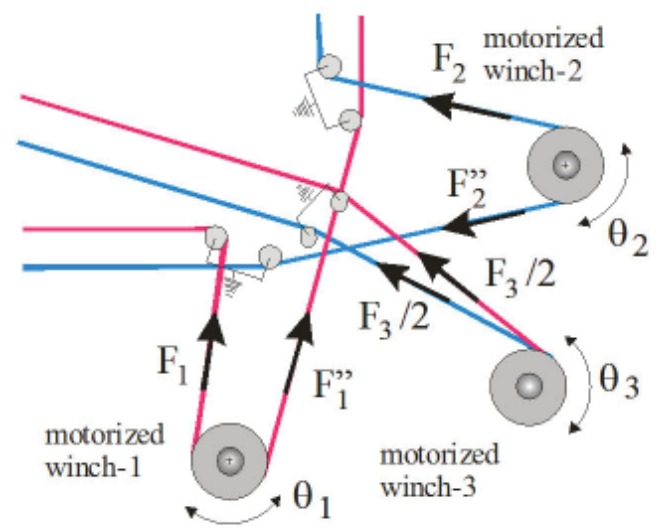

a)

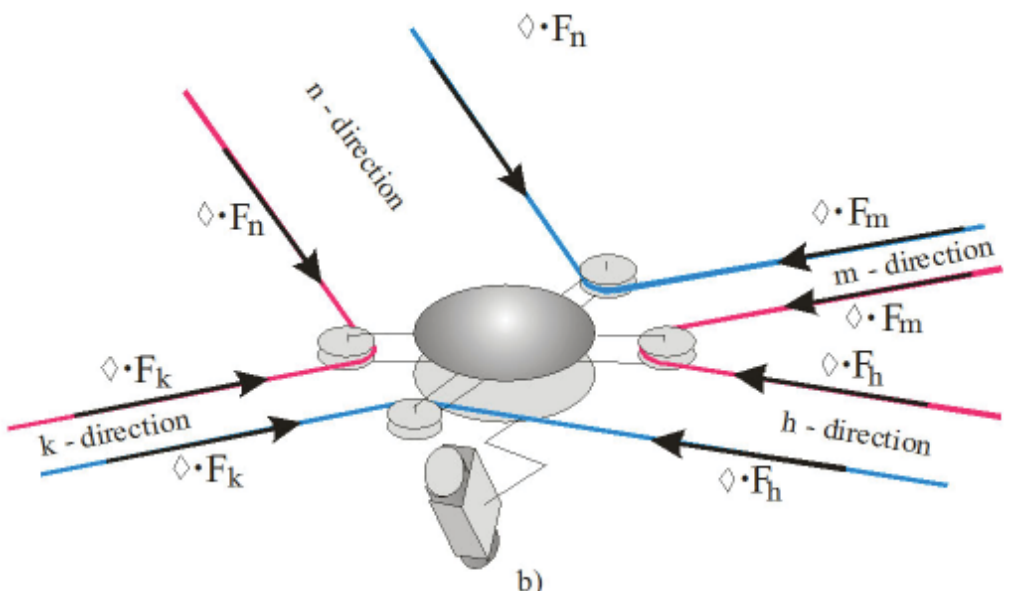

b)

Figure 3. a) The motors (ropes) forces before motor 1, motor 2 and motor 3 and after motor 1 and motor 2 , b) The ropes forces which carry a camera

By differentiating equations (1)-(4) and substituting them into the equations (10)-(12), the relationship between velocities of camera carrier coordinates $\overrightarrow{\dot{p}}=\left[\begin{array}{lll}\dot{x} & \dot{y} & \dot{z}\end{array}\right]^{T}$ and velocities of motor coordinates $\overrightarrow{\dot{\phi}}=\left[\begin{array}{lll}\dot{\theta}_{1} & \dot{\theta}_{2} & \dot{\theta}_{3}\end{array}\right]^{T}$ can been obtained:

$$
\overrightarrow{\dot{\phi}}=J_{a} \cdot \overrightarrow{\dot{p}}
$$

The acceleration vector $\overrightarrow{\ddot{\phi}}$ can be defined by using Newton Euler method.

Jacobian matrix $J_{a}$ is a full matrix. The elements of Jacobian matrix that are beyond the diagonal $J_{a 12}, J_{a 13}, J_{a 21}, J_{a 23}, J_{a 31}, J_{a 32}$, are not equal to zero and show the strong coupling between the velocities of camera carrier coordinates and velocities of motor coordinates.

This methodology for developing the kinematic model of selected CPR-A systems is named the KinCPR-Solver (Kinematic Cable Parallel Robot Solver), and it gives a precise direct and inverse kinematic solutions.

The mathematical model of the system has the following form:

$$
\vec{u}=G_{v} \cdot \ddot{\phi}+L_{v} \cdot \dot{\phi}+S_{v} \cdot \vec{M}_{a}
$$

Where: $\vec{u}=\left[\begin{array}{lll}u_{1} & u_{2} & u_{3}\end{array}\right]^{T}, G_{v}=\operatorname{diag} G_{v i} L_{v}=\operatorname{diag} L_{v i^{\prime}}$ $S_{v}=$ diag $_{v i{ }^{\prime}}$ Vector equation (14) is given by applying Lagrange's equation on the generalized coordinates $\theta_{1}, \theta_{2}, \theta_{3}$.

Lagrange's principle of virtual work has been used to find the relation between the resultant motor load moment $M_{a}$ and the camera carrier force $\vec{F}$.

$$
\vec{M}_{a}=\left(\left(J_{a}\right)^{T}\right)^{-1} \cdot \vec{F}
$$

The system has two ropes in the each direction and the motor 3 simultaneously unwind or wind both ropes. The connection between the resultant motor load moment $\vec{M}_{a}$ and camera carrier force $\vec{F}$ is established. See equation (16).

$\vec{M}_{a}=O_{a} \cdot \vec{F}$

By substituting equation (16) into the equation (14), the dynamic model of the CPR-A system has been generated:

$\vec{u}=G_{v} \cdot \overrightarrow{\ddot{\phi}}+L_{v} \cdot \overrightarrow{\dot{\phi}}+S_{v} \cdot O_{a} \cdot \vec{F}$

The moment mapping matrix $\mathrm{O}_{a}$ indicates that the system is highly coupled.

Control law is selected by the local feedback loop for the position and velocity of the motor shaft in the following form:

$$
\vec{u}_{i}=K_{l p i} \cdot\left(\vec{\theta}_{i}^{o}-\vec{\theta}\right)+K_{i} \cdot\left(\overrightarrow{\dot{\theta}}_{i}^{o}-\overrightarrow{\dot{\theta}}_{i}\right)
$$

Where is position constant $K_{l p i}$, and velocity constant $K_{k v i}$ for the motion control.

\section{SIMULATION RESULTS AND ANALYSES}

The CPR-A system presented in Figure 1 and 2 is modeled and analyzed by the software package AIRCAMA. The software package AIRCAMA is used for validation of the applied theoretical contributions.

The software package AIRCAMA includes three essential modules, which are the kinematic, dynamic and motion control law solvers for the CPR-A system.

The most important element of the CPR-A system is the motor mathematical model which is an integral part of software package AIRCAMA. 
Through the simulation results it is shown that the dynamic characteristics of the motor significantly affect the response of the system and its stability.

The camera carrier motion dynamics directly depends of the mechanism dynamic parameters. The CPR-A model has been analyzed using the trajectory and the selected system parameters. In order to make the results comparable, the simulation is made for the same desired system parameters defined in the Nomenclature. The motors are selected by Heinzman SL100F and the gear boxes are HFUC14-50-2A-GR+belt.

The system is coupled on the reference level and its mathematical model is defined by the equations (1)-(17).

The camera moves in 3D space ( $x, y$ and $z$ directions). The camera carrier has the starting point $p^{0}{ }_{\text {start }}=\left[\begin{array}{lll}0.7 & 1.0 & -0.1\end{array}\right](\mathrm{m})$, and the end point $p_{\text {end }}^{0}=\left[\begin{array}{lll}1.3 & 1.6 & -0.8\end{array}\right](\mathrm{m})$ (see Figure 4a)). The camera motion velocity has a trapezoid form and $p_{\text {max }}^{0}=0.417(\mathrm{~m} / \mathrm{s})$ as shown in Figure $\left.4 \mathrm{~b}\right)$.
All three motor's angular positions $\theta_{1}, \theta_{2}$ and $\theta_{3}$ are involved in the coordinated task generation. This clearly represents a proof that all of these motions are mutually coupled.

The system responses, for first and second Examples are shown in Fig. 5 and 6, respectively. These results are comparable and therefore are shown in Table 1.

Each example has six pictures related to the:

a) camera carrier position at the reference and the real frames,

b) motor shaft position at the reference and the real frames,

c) motors forces, forces in the ropes at the reference and the real frames,

d) deviation between the real and the reference trajectory of the camera carrier,

e) deviation between the real and the reference trajectory of the motor shaft positions,

f) control signals at the reference and the real frames.

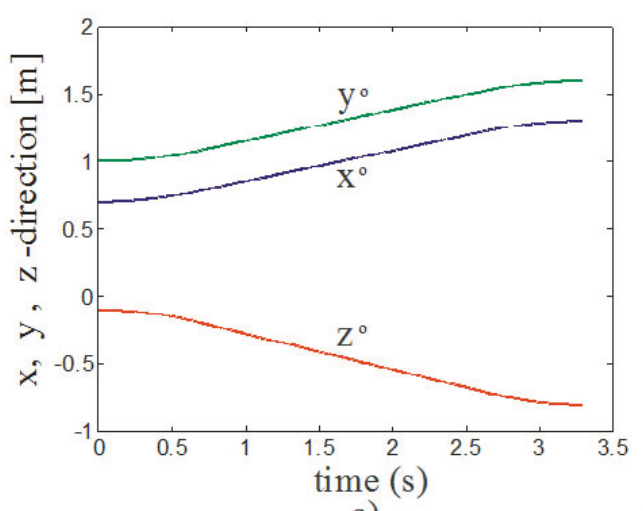

a)

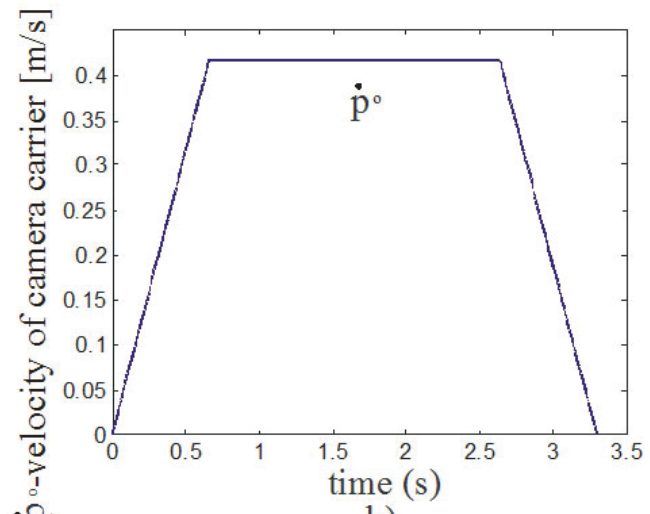

b)

Figure 4: The reference trajectory motion of a) position $x^{0}, y^{0}, z^{0}$ b) velocity $p_{\text {max }}^{0}=0.417(\mathrm{~m} / \mathrm{s})$ of camera carrier

Table 1: Comparison of two selected Examples

\begin{tabular}{|c|c|c||c|c|}
\hline Example & Figure & $p_{\text {max }}^{0}(m / s)$ & winch radius $R_{i}(m)$ & $\begin{array}{c}\text { motors reach } \\
\text { saturation }\end{array}$ \\
\hline \hline 1 & 5 & \multirow{3}{*}{0.417} & $R_{1}=R_{2}=R_{3}=0.15(\mathrm{~m})$ & no \\
\hline 2 & 6 & & $R_{1}=0.075(\mathrm{~m}), R_{2}=0.04(\mathrm{~m}), R_{3}=0.06(\mathrm{~m})$ & yes, all 3 motors \\
\hline
\end{tabular}

\section{A. Example 1}

The first Example is analyzing the CPR-A system which parameters are presented in the Nomenclature Section. This system is working under the ideal conditions. See Figure 5.

B. Example 2

The second Example shows the response of the CPR-A system, which differs in the radius of all three motorized pulleys in comparison to the Ex- ample 1. Other system parameters are the same as in Example 1. For the chosen smaller pulleys radii the motors speed must be higher to achieve the desired trajectory of the camera carrier which is predefined to be the same like in the Example 1. See Figure 6.

The CPR-A system being developed in the Mihajlo Pupin Institute, which observes space, is a part of a more complex system. 


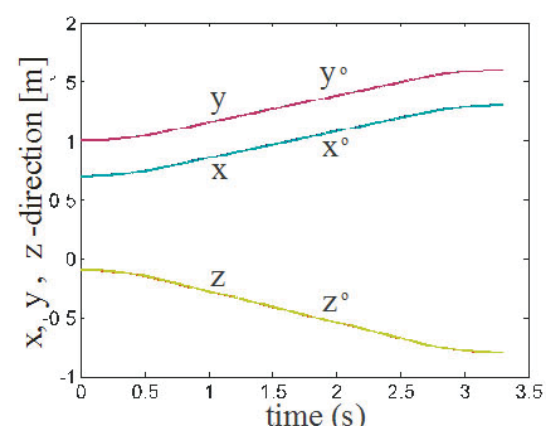

a)

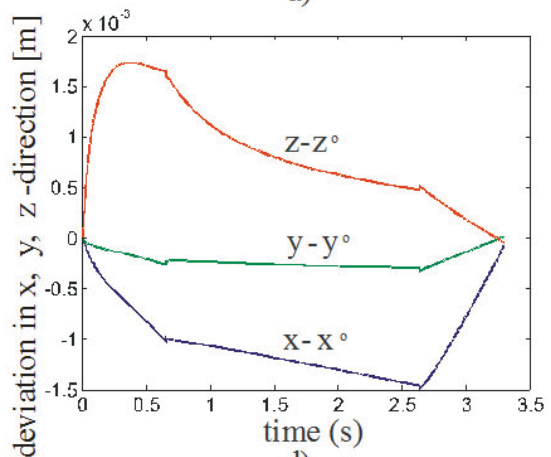

d)

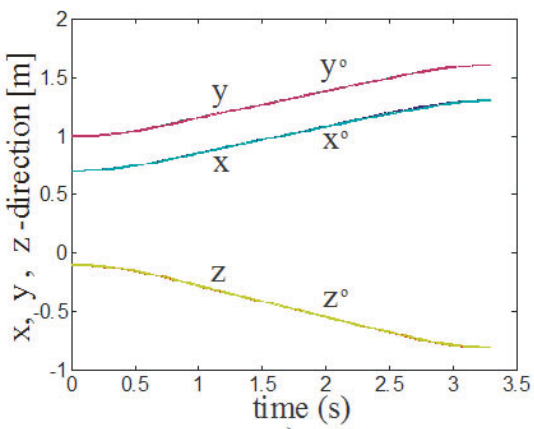

a)

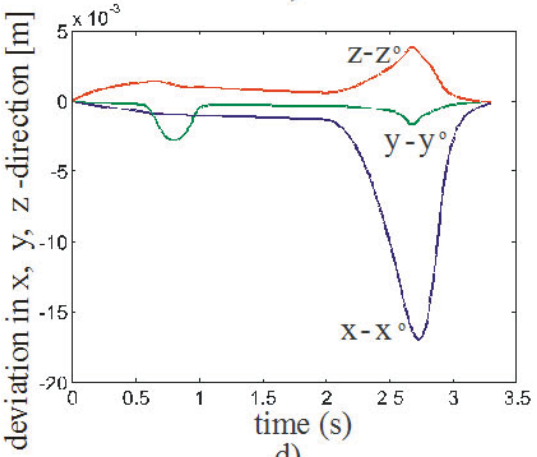

d)

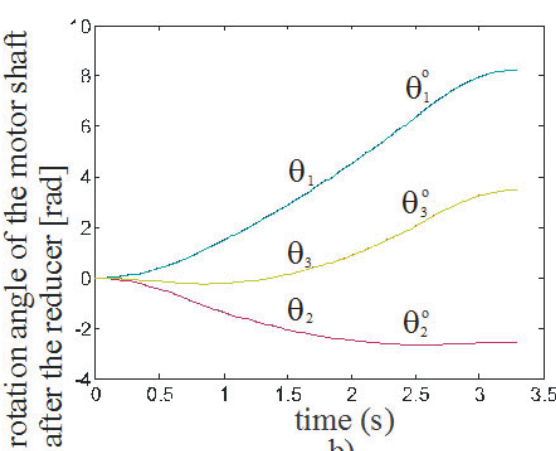

b)

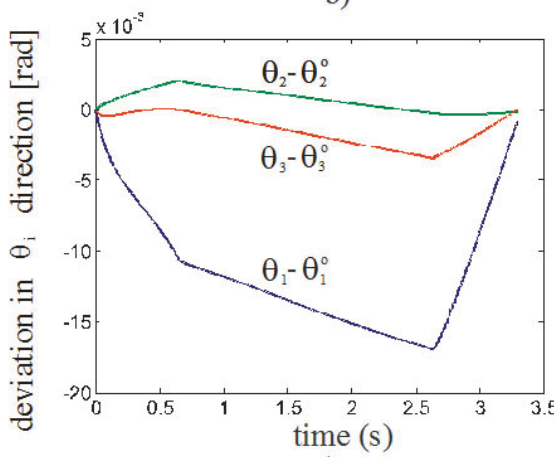

e)

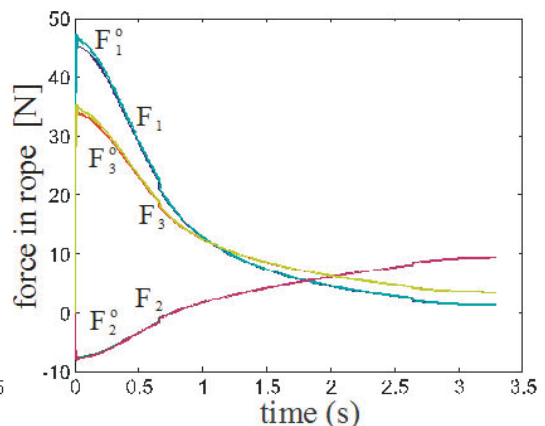

c)

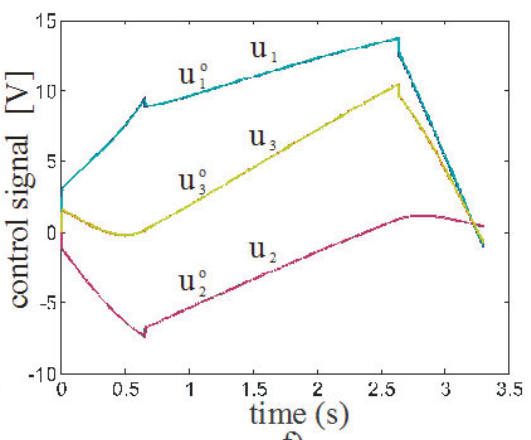

f)

Figure 5: Example 1

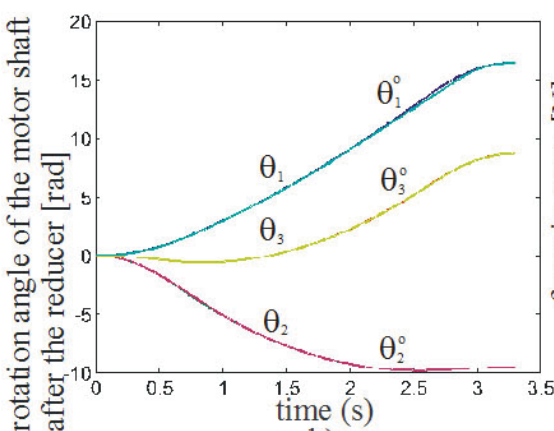

b)

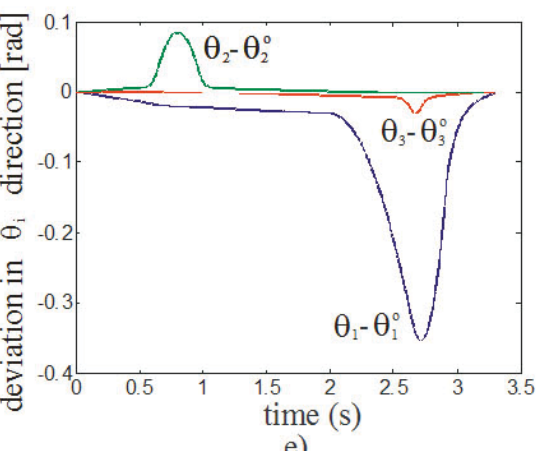

e)

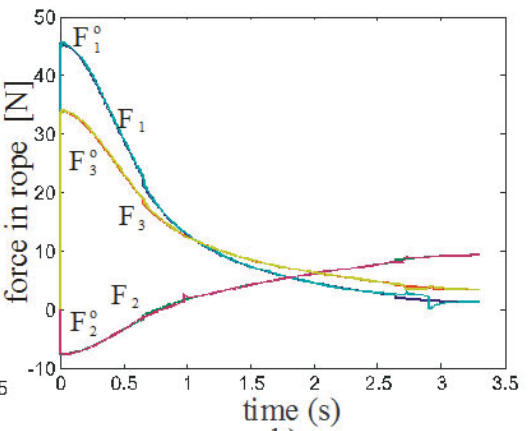

b)

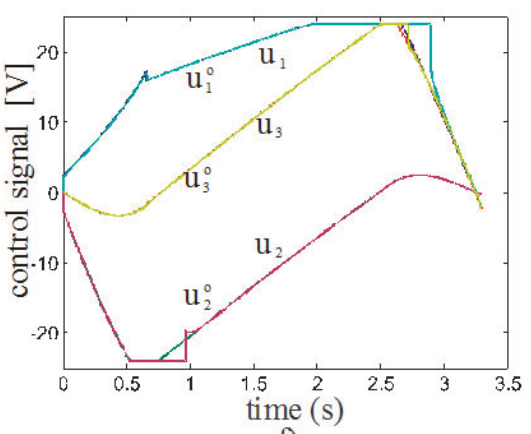

f)

Figure 6: Example 2

\section{CONCLUSION}

The mathematical model of the CPR-A system is defined in the first phase of the study in this paper. It shows the importance of generating the Jacobian matrix that defines the kinematic model of the CPR-A system. The relation between the camera carrier motion and the motors angular positions has been established. This novel pro- cedure is named KinCPR-Solver which means Kinematic Cable Parallel Robot Solver.

The Jacobian matrix plays an important role in developing the CPR-A dynamic model. The Lagrange's principle of virtual work has been used for solving the complex relation between the resultant motor load moment (acting as a load at the first, second and third motor shaft) and camera carrier forces (acting at the camera carrier). 
This relation is used for generating the dynamic model of this system.

A very important feature of the coupling between the motion of the camera carrier in the space of the Cartesian coordinates and the motion of motors is discovered. The results show how significant it is to know the properties of the coupling and its effects on the accuracy of the camera carrier motion. The selected characteristics of the motors are satisfactory in the considered examples. The AIRCAMA software package can be used for the confirmation of the validity of defined mathematical models and for the analysis and selection of all parameters of the CPR-A system also. Two different examples have been presented to show the CPR-A application and validity. For future research it is planned to design and test few control algorithms based on papers [25] and [21]. Also, the influence of disturbance force on motion of the camera carrier is going to be analyzed in future [24].

\section{NOMENCLATURE}

DOF - Degree Of Freedom

CPR - Cable-suspended Parallel Robot

AIRCAMA - software packages AIRCAMA (aerial camera system type A)

$t(s)$ - time

$d_{t}=0.0001(\mathrm{~s})$ - sample time

Camera carrier coordinate (position of camera carrier in the Cartesian space): $\vec{p}=\left[\begin{array}{lll}x & y & z\end{array}\right]^{T}[m]$ Total number of DOF: $i=1,2,3$.

Vector of motor coordinates: $\vec{\phi}=\left[\begin{array}{lll}\theta_{1} & \theta_{2} & \theta_{3}\end{array}\right]^{T}[\mathrm{rad}]$

Motor shaft angular position after the gear box:

$\theta_{i}\left(\theta_{i}^{o}\right)[\mathrm{rad}]$

Motor force, resultant force:

$\vec{F}_{a}=\left[\begin{array}{lll}F_{1} & F_{2} & F_{3}\end{array}\right]^{T}[N]$

Acting force on the camera carrier:

$\vec{F}_{p}=\left[\begin{array}{lll}F_{p x} & F_{p y} & F_{p z}\end{array}\right]^{T}[N]$

Camera carrier force, total force at the camera carrier: $\vec{F}=\left[\begin{array}{lll}F_{x} & F_{y} & F_{z}\end{array}\right]^{T}[N]$

Resultant motor load moment:

$\vec{M}_{a}=\left[\begin{array}{lll}M_{1} & M_{2} & M_{3}\end{array}\right]^{T}[\mathrm{Nm}]$

Winch radius: $R_{i}=0.15(\mathrm{~m})$

Jacobian matrix: $J_{a}$

Rotor circuit resistance: $R_{r i}=0.917(\Omega)$
Voltage: $u_{i}(V)$

Electromotive force constant: $i_{i}[A]$

Rotor current:

$C_{E i}=3.3942[\mathrm{~V} /(\mathrm{rad} / \mathrm{s})]$

Constant of the moment proportionality:

$C_{M i}=2.5194[\mathrm{Nm} / \mathrm{A}]$

Coefficient of viscous friction:

$B_{C i}=0.0670[\mathrm{Nm} /(\mathrm{rad} / \mathrm{s})]$

Moment of inertia for the rotor and the gear box: $J_{r i}=1.5859\left[\mathrm{kgm}^{2}\right]$

Motor inertia characteristic:

$G_{v i}=\frac{J_{r i} \cdot R_{r i}}{C_{M i}}=0.1787$

Motor damping characteristic:

$L_{v i}=\frac{R_{r i} \cdot B_{C i}}{C_{M i}}+C_{E i}=3.4186$

Motor geometric characteristic:

$S_{v i}=\frac{R_{r i}}{C_{M i}}=0.364$

Mass of the camera carrier: $m=1[\mathrm{~kg}]$

Moment mapping matrix: $O_{a}(m)$

Length of the recorded field: $d=3.2[\mathrm{~m}]$

Width of the recorded field: $s=2.2[\mathrm{~m}]$

Height of the recorded field: $v=2.0[\mathrm{~m}]$

Initial deviation of the motor angular position:

$\delta \theta_{i}\left(t_{o}\right)=0(\mathrm{rad}), \delta \dot{\theta}_{i}\left(t_{o}\right)=0[\mathrm{rad} / \mathrm{s}]$

Positional constant for motion control:

$K_{l p}=\left[\begin{array}{lll}800 & 3500 & 3000\end{array}\right]$

Velocity constant for motion control:

$K_{l v}=\left[\begin{array}{lll}60 & 120 & 110\end{array}\right]$

\section{ACKNOWLEDGMENT}

This research has been supported by the Ministry of Education, Science and Technological Development, Government of the Republic of Serbia Grant TR-35003 through the following two projects: "Ambientally intelligent service robots of anthropomorphic characteristics", by Mihajlo Pupin Institute, University of Belgrade, Serbia, Grant Ol-174001 and "The dynamics of hybrid systems of complex structure", by Institute SANU Belgrade and Faculty of Mechanical Engineering University of Nis, Serbia, and partially supported by the project SNSF Care-robotics project no. IZ74Z0-137361/1 by Ecole Polytech- 
nique Federale de Lausanne, Switzerland. We are grateful to Prof. Dr. Katica R. (Stevanovic) Hedrih from Mathematical Institute, Belgrade for helpful consultations during the implementation of this paper.

\section{REFERENCES}

1) Borgstrom, P., H., Borgstrom, N., P., Stealey, M., J., Jordan, B., Sukhatme, G., Batalin, M., A., Kaiser, W., J., (2007) Discrete Trajectory Control Algorithms for NIMS3D, an Autonomous Underconstrained Three-Dimensional Cabled Robot, Proceedings of the 2007 IEEE/ RSJ International Conference on Intelligent Robots and Systems, San Diego, CA, USA.

2) Bruckmann, T., Mikelsons, L., Schramm, D., Hiller, M., (2007) Continuous workspace analysis for parallel cable-driven StewartGough platforms", Special Issue: Sixth International Congress on Industrial Applied Mathematics (ICIAM07) and GAMM Annual Meeting, Zürich, 7(1)

3) Carricato, M., (2011) Under-constrained cable-driven parallel robots, part of book Quarta giornata di studio Ettore Funaioli - 16 luglio 2010, Asterisco, 443-454.

4) Đurić, A., Saidi, R., Al, ElMaraghy, W., (2012) Dynamics Solution of n-DOF Global Machinery Model, Robotics and Computer Integrated Manufacturing (CIM) Journal, 28(5), 621-630.

5) Đurić, A., M., Saidi, R., Al, ElMaraghy, W., H., (2010) Global Kinematic Model Generation for n-DOF Reconfigurable Machinery Structure, 6th IEEE Conference on Automation Science and Engineering, CASE 2010, Toronto, Canada.

6) Dondur N., Spasojević-Brkić, V., Brkić, A., (2012) Crane cabins with integrated visual systems for detection and interpretation of environment - economic appraisal, Journal of Applied Engineering Science (Istraživanja i projektovanja za privredu), No. 4, Vol. 10, pp. 191-196.

7) Duan, B.,Y., (1998) A new design project of the line feed structure for large spherical radio telescope and its nonlinear dynamic analysis, Mechatronics, 9, 53-64.

8) Fang, S., Franitza, D., Torlo, M., Bekes, F., Hiller, M., (2004) Motion control of a tendonbased parallel manipulator using optimal tension distribution, IEEE/ASME Trans. Mechatron., 9(3), 561-568.
9) Filipovic, M., Potkonjak, V., Vukobratovic, M., (2007) Humanoid robotic system with and without elasticity elements walking on an immobile/mobile platform, J. Intell. Robot. Syst. 48, 157-186.

10) Filipovic, M., Vukobratovic, M., (2008) Complement of Source Equation of Elastic Line, Journal of Intelligent \& Robotic Systems, International Journal, 52(2), 233-261.

11) Filipovic, M., Vukobratovic, M., (2008) Expansion of source equation of elastic line, Robotica, Cambridge University Press, 1-13.

12) Filipovic, M., (2012) Relation between EulerBernoulli Equation and Contemporary Knowledge in Robotics, Robotica, 1-13.

13) Gosselin, C., Grenier, M., (2011) On the determination of the force distribution in overconstrained cable-driven parallel mechanisms, Meccanica, An International Journal of Theoretical and Applied Mechanics, Springer, 46 (1), 3-15.

14) Gosselin, C., Ren, P., Foucault, S., (2012) Dynamic Trajectory Planning of a Two-DOF Cable-Suspended Parallel Robot, 2012 International Conference on Robotics and Automation RiverCentre, Saint Paul, Minnesota, USA, May 14-18, 2012.

15) Gouttefarde, M., Merlet, J-P., Daney, D., (2006) Determination of the wrench-closure workspace of 6-DOF parallel cable-driven mechanisms, Advances in Robot Kinematics, 5, 315-322.

16) Hedrih, (Stevanovic), K., (2010) Energy analysis in the hybrid system forced regimes, Proceeding of Institute of Mathematics NANU Ukraine, 7(3), 90-107.

17) Hedrih, (Stevanovic), K., (2012) Energy and Nonlinear Dynamics of Hybrid System, Chapter in Book Dynamical Systems and Methods, Edited by Albert Luo, Springer, 1, 29-83.

18) Higuchi, T., Ming, A., .Jiang-Yu, J., (1988) Application of multi-dimensional wire crane in construction, In 5th Int. Symp. on Robotics in Construction, Tokyo, June, 6-8, 661-668.

19) Hiller, M., Fang, S., Q., (2005) Design, analysis and realization of tendon-base parallel manipulators, Mech. Mach. Theory, 40, 429-445.

20) Kozak, K., Zhou Q., J., Wang, J., (2006) Static Analysis of Cable-Driven Manipulators With Non-Negligible Cable Mass, IEEE Transaction on Robotics, 22(2), 425-433. 
21) Lazarevska, M., Trombeva-Gavrilovska, A., Knežević, M., Samardžioska, T., Cvetkovska, M. (2012) Neural network prognostic model for rc beams strenghtened with CFRP strips, Journal of Applied Engineering Science (Istraživanja i projektovanja za privredu), No. 1, Vol. 10, pp. 27-30.

22) Merlet, J., P., (2010) MARIONET, A Family of Modular Wire-Driven Parallel Robots, Advances in Robot Kinematics: Motion in Man and Machine, 1, 53-61.

23) Miermeister, P., Pott, A., Verl, A., (2012) AutoCalibration Method for Overconstrained Cable-Driven Parallel Robots, ROBOTIK 2012 - 7th German Conference on Robotics, Munich, Germany.

24) Mijailović, R., (2012) Methodology for estimating the dependence between force and deplacement - a vehicle crash case, Journal of Applied Engineering Science (Istraživanja i projektovanja za privredu), No. 1, Vol. 10, pp. 1-8.

25) Nunes, I., (2012) Fuzzy systems to support industrial engineering management, Journal of Applied Engineering Science (Istraživanja i projektovanja za privredu), No. 3, Vol. 10, pp. 143-146.

26) Oh, S-R., Agrawal, S., K., A., (2005) Reference Governor-Based Controller for a Cable Robot Under Input Constraints, IEEE Transaction on Control Systems Technology, 13(4), 639-645.

27) Pott, A., (2008) Forward Kinematics and Workspace Determination of a Wire Robot for Industrial Applications, Advances in Robot Kinematics: Analysis and Design, 7, 451-458.

28) Raskovic, D., (1965) Theory of oscillations, Scientific book, Belgrade, Serbia.
29) Rega, G., (2004) Nonlinear vibrations of suspended cables-Part I: modeling and analysis, ASME, APPL MECH REV, 57(6), 443-478.

30) Rega, G., (2004) Nonlinear vibrations of suspended cables-Part II: deterministic phenomena, ASME, APPL MECH REV, 57(6), 479-514.

31) Shiang, W-J., Cannon, D., Gorman, J., (2000) Optimal Force Distribution Applied to a Robotic Crane with Flexible Cables, Proceedings of the 2000 IEEE International Conference on Robotics \& Automation, San Francisco, Ca, April 2000, 1948-1954.

32) Su, X., Y., Duan, B., Y., (2000) The Application of the Stewart Platform in Large Spherical Radio Telescopes, Journal of Robotic Systems by John Wiley \& Sons, 17(7), 375-383.

33) Su, X., Y., Duan, B., Y., (2000) The mathematical design and kinematics accuracy analysis of a fine tuning stable platform for the large spherical radio telescope, Mechatronics, 10, 819-834.

34) Yao, R., Tang, X., Wang, J., Huang, P., (2010) Dimensional Optimization Design of the Four-Cable Driven Parallel Manipulator in FAST, IEEE/ASME Transaction on Mechatronics, 15(6) 932-941.

35) Zi, B., Duan, B., Y., Du, J., L., Bao H., (2008) Dynamic modeling and active control of a cable-suspended parallel robot, Mechatronics, 18, 1-12.

Paper sent to revision: 16.10.2013.

Paper ready for publication: 11.12.2013. 